\title{
CERTAIN MAPPINGS OR DECOMPOSITIONS WHICH ARE TOPOLOGICALLY PROJECTIONS
}

\author{
BY LOUIS F. MCAULEY 1
}

Communicated by Richard Anderson, January 20, 1969

Introduction. A general question which is of interest is the following. Suppose that $f$ is a mapping of a compact metric continuum $X$ onto a metric space $Y$. Under what conditions is there an embedding of $X$ and $Y$ in $E^{n}$ (Euclidean $n$-space) or $H^{\omega}$ (Hilbert space) so that $f$ is topologically equivalent to a projection onto $Y$ defined by some collection of parallel hyperplanes? Theorem 1 below provides an answer for a very special case of this general question. Although this theorem is actually a corollary of a more general theorem, we feel that its proof provides motivation and understanding for the main theorem.

TheOREM 1. Suppose that $U$ is the Universal 1-dimensional Menger Curve [1] and that $f$ is a light open mapping of $U$ onto $I$ (the interval $[0,1])$ such that $f^{-1}(x)$ is homeomorphic to a Cantor set for each $x$ in $I$. Then there is a homeomorphism $h$ of $U$ into $E^{3}$ such that the mapping $p$ defined by projecting $U$ onto I through planes parallel to the yz-plane is topologically equivalent to $f$, that is, $p h=f$.

We shall sketch a proof of this theorem. Our proof depends on an important theorem of J. H. Roberts [5] concerning contractibility in spaces of homeomorphisms, some very useful techniques of Dyer and Hamstrom [2], and a powerful selection theorem of E. A. Michael [4].

Statements of some results used in our proofs. Suppose that $X$ is a compact metric space and dimension $X=n$ (an integer). For each positive integer $k$, let $H\left(X, I^{k}\right)$ be the space of all homeomorphisms of $X$ into $I^{k}$ (a $k$-cell) and let $C\left(X, I^{k}\right)$ be the space of all mappings of $X$ into $I^{k}$. The metric, in each case, is the usual one: $\rho(f, g)=$ $\max d(f(x), g(x))$ for $x$ in $X$ and $d$ is the usual metric for $I^{k}$.

Theorem (J. H. Roberts [5]). Suppose that each of $X$ and $K$ is a compact metric space, $\operatorname{dim} X=n, \operatorname{dim} K=r$, and $k \geqq 2 n+2+r$. Let $\alpha_{0}$ and $\alpha_{1}$ be mappings of $K$ into $C\left(X, I^{k}\right)$. Then there exists a homotopy $f: K \times I \rightarrow C\left(X, I^{k}\right)$ such that

(1) $f(\omega, 0)=\alpha_{0}(\omega), f(\omega, 1)=\alpha_{1}(\omega), \omega \in K$, and

(2) for each $t, 0<t<1, f(\omega, t) \in H\left(X, I^{k}\right)$.

\footnotetext{
1 Research supported in part by NSF Grant GP 6951.
} 
Theorem (E. A. Michael [4]). If each of $A$ and $B$ is a metric space, $A$ is complete, covering dimension of $B \leqq n+1, Z$ is a closed subset of $B, F$ is a function taking $A$ onto $B$ such that the collection of inverses under $F$ is lower semicontinuous (defined below) and equi-LCn (as defined below), and $f$ is a mapping of $Z$ into $A$ such that for $z$ in $Z$, $f(z) \in F^{-1}(z)$, then there is a neighborhood $U$ of $Z$ in $B$ such that $f$ can be extended to a mapping $f^{*}$ of $U$ into $A$ such that for $b \in U, f^{*}(b) \in F^{-1}(b)$. If each inverse under $F$ has the property that its homotopy groups of order $\leqq n$ vanish, then $U$ may be taken to be the space $B$.

Notation and definitions. In this paper, all mappings are continuous and all spaces are metric. A mapping $f$ of a space $X$ into a space $Y$ is light iff $f^{-1} f(x)$ is totally disconnected for each $x$ in $X$. And, $f$ is open iff for each $U$ open in $X, f(U)$ is open relative to $f(X)$. A characterization of the Universal 1-dimensional Curve $U$ may be found in R. D. Anderson's paper [1].

Definition (Dyer aNd Hamstrom [2]). A mapping $p: T \rightarrow B$ is said to be completely regular iff for each $\epsilon>0$ and each point $b$ in $B$, there is a $\delta>0$ such that if $x \in B$ and $d(x, b)<\delta$, then there exists a homeomorphism $h_{b x}$ of $p^{-1}(b)$ onto $p^{-1}(x)$ which moves no point as much as $\epsilon$.

Definition. A collection $G$ of closed point sets filling a metric space $X$ (i.e., the union of the elements of $G$ is $X$ ) is said to be equi$L C^{n}$ iff for each $\epsilon>0, g$ in $G$, and $x \in g$, there is a $\delta>0$ such that if $h \in G$ and $f$ is a mapping of a $k$-sphere $S^{k}, 0 \leqq k \leqq n$ into $h \cap N_{\delta}(x)$, then there is an extension $F$ of $f$ to the $(k+1)$-disk $D^{k+1}$, into $h \cap N_{\epsilon}(x)$.

The hypothesis of Theorem 1 is not vacuously satisfied. Such mappings are easy to construct.

Indication of a proof of Theorem 1. Let $A$ denote a unit cube (3-cell) in $E^{3}$ whose vertices are $(0,0,0),(0,0,1),(0,1,0),(1,0,0)$, $(1,0,1),(0,1,1),(1,1,0)$, and $(1,1,1)$. Let $C_{x}$ denote a 2 -cell section of $A$ cut by the plane perpendicular to the $x$-axis at $x$.

For each $x$, let $H\left(f^{-1}(x), C_{x}\right)$ denote the space of all homeomorphisms of $f^{-1}(x)$ into $C_{x}$. For convenience, we shorten this to $H_{x}$. We use the usual metric on $H_{x}$, i.e., for $g, h$ in $H_{x}, \rho(g, h)=\max \{\rho[g(x), H(x)]\}$. Now, $H_{x}$ is a topologically complete metric space.

Consider the collection $H$ of all $H_{x}$ and let $H^{*}$ denote the union of the elements of $H$. The space $H^{*}$ is a topologically complete metric space. This follows from a theorem in [3]. However, we shall indicate here how a metric may be defined.

A metric for $H^{*}$. If $g \in H^{*}$, then $g \in H_{x}$ for some $x$. Let $\hat{g}$ denote 
the graph of $g$ in $U \times C$ where $C$ is a 2-cell. For each pair of elements $g, h$ of $H^{*}$ where $g \in H_{x}$ and $h \in H_{y}$, let $D(g, h)=$ Hausdorff distance between $\hat{g}$ and $\hat{h}$. Although $D$ is a metric for $H^{*}$, it may not be complete. However, $H^{*}$ is a topologically complete metric space. This follows from Theorem 1 of [3].

By a theorem of Roberts [5], $H_{x}$ for each $x$ is locally connected. The collection $H$ of all $H_{x}$ is equi-locally connected in the homotopy sense (equi- $L C^{0}$ ). That is, for each $H_{x}, p \in H_{x}$, and $\epsilon>0$, there is a $\delta>0$ such that if $\phi$ is a mapping of $S^{0}$ (a 0 -sphere or pair of points) into $N_{\delta}(p) \cap H_{\nu}$ for $H_{\nu}$ in $H$, then $\phi$ can be extended to a mapping $\Phi$ which takes a 1-cell into $N_{\epsilon}(p) \cap H_{y}$. This may be proved by first showing that $f$ is actually completely regular. Next, apply an argument similar to Dyer and Hamstrom [2] or to mine in [3].

Let $H^{*}$ be the union of the elements of $H$ and $F$ denote the function from $H^{*}$ onto $I=[0,1]$ such that $F^{-1}(x)=H_{x}$. It follows that $F$ is lower semicontinuous. That is, if $\left\{h_{i}\right\} \rightarrow h$ where $h_{i}, h \in H$, then $H_{h}$ is in the closure of $\bigcup_{i=1}^{\infty} H_{h_{i}}$. See [3, p. 137]. Now by a selection theorem of Michael [4], there is a continuous selection $\Phi$ from an open interval $(a, b)$ to $H^{*}$ such that $\Phi(x) \in F^{-1}(x)=H_{x}$. By Corollary 2 of [5], $F^{-1}(x)$ for each $x$ in $I$ is arcwise connected. Thus, by Michael's Theorem [4, p. 563], $(a, b)$ may be taken as the space $[0,1]$. The mapping $\Phi$ induces a homeomorphism $h$ from $f^{-1}[0,1]=U$ into $A$ (a 3-cell) such that $h \mid f^{-1}(x)=\Phi(x)$. That is, for $u$ in $U$, $h(u)=\Phi[f(u)](u)$. It follows that $f=p h$ where $p$ is the projection of $A$ onto $I$ by planes parallel to the $y z$-plane. The theorem is proved.

REMarks. Projections need not be local products (locally trivial fiber spaces), even in the case that $p: X \rightarrow Y$ has the property that all sets $p^{-1} p(x)$ are homeomorphic for the various $x \in X, X$ is a Peano continuum, $p$ is open, and $p$ is monotone. See Ungar's example [6].

Main theorem. Now, we are ready to state the general theorem for which Theorem 1 is a special case.

Theorem 2. Suppose that $f: X \Rightarrow I^{r+1}$ is a completely regular mapping, $X$ is a complete metric space, for each $x$ in $X, f^{-1} f(x) \cong K, a$ compact $n$-dimensional set. Let $k \geqq 2 n+2+r$. Then there is a homeomorphism $h$ of $X$ into $I^{k+r+1}$ such that $f=p h$ where $p$ is the projection mapping of $I^{k} \times I^{r+1}$ onto $I^{r+1}$.

It should be clear from the indicated proof of Theorem 1 that a similar argument yields Theorem 2 . 


\section{BIBLIOGRAPHY}

1. R. D. Anderson, A characterization of the universal curve and a proof of its homogeneity, Ann. of Math. (2) 67 (1958), 313-324.

2. Eldon Dyer and M. E. Hamstrom, Completely regular mappings, Fund. Math. 45 (1957), 103-118.

3. L. F. McAuley, The existence of a complete metric for a special mapping space and some consequences, Ann. of Math. Studies, no. 60, Princeton Univ. Press, Princeton, N. J., 1966, pp. 135-139.

4. E. A. Michael, Continuous selections. I, II, III, Ann. of Math. (2) 63 (1956), 361-382; (2) 64 (1956), 562-580; (2) 65 (1957), 357-390.

5. J. H. Roberts, Contractibility in spaces of homeomorphisms, Duke Math. J. 28 (1961), 213-220.

6. Gerald S. Ungar, A pathological fiber space, Illinois J. Math. 12 (1968), 623-625.

Rutgers University, New Brunswick, New Jersey 08903 